\title{
THYROID FUNCTION
}

\section{AUTOIMMUNITY IN}

\section{PREGNANGY AND ADHD}

High maternal levels of thyroid peroxidase antibodies (TPOAb) during early pregnancy are associated with externalizing behavioral problems in children aged 3 years, report researchers from the Generation R Study.

A link between maternal thyroid dysfunction during pregnancy and the risk of cognitive and behavioral problems in children has previously been reported, but the implication of maternal thyroid autoimmunity in the development of these problems is less clear.

The Generation R Study involves a large cohort of individuals from Rotterdam, The Netherlands. Ghassabian and colleagues analyzed data from 3,139 children and their mothers. The researchers evaluated measures of maternal thyroid function (TSH and free $\mathrm{T}_{4}$ levels) and thyroid autoimmunity (TPOAb levels) at $13.5 \pm 1.8$ weeks of gestation. They then investigated the presence of verbal and nonverbal cognitive problems when the children were aged 2.5 years and the presence of externalizing and internalizing behavioral problems in children aged 3 years, on the basis of standardized surveys filled in by the children's parents.

Among the pregnant women who enrolled in the study, 147 had TPOAb levels $>100 \mathrm{IU} / \mathrm{ml}$ and were classified as TPOAb-positive. In contrast to previous findings, verbal and nonverbal cognitive delay in children (assessed on the basis of the Language Development Survey and the Parent Report of Children's Ability, respectively) were not associated with the presence of high TPOAb levels in mothers. However, the risk of attention deficit/hyperactivity disorder (ADHD), as assessed by the Child Behavior Checklist $1 \frac{1}{2}-5$, was significantly higher in children whose mothers were TPOAbpositive (OR 1.77, 95\% Cl 1.15-2.72, $P=0.01$ ), independently of TSH levels.

The researchers point out that the mechanisms underlying this relationship need to be investigated further. They also comment that the implications of the findings for clinical practice are not clear, but suggest that TPOAb-positive women and their children might benefit from closer monitoring and early detection of developmental delay.

Joana Osório

Original article Ghassabian, A. et al. Maternal thyroid autoimmunity during pregnancy and the risk of attention deficit/hyperactivity problems in children. The Generation $\mathrm{R}$ Study. Thyroid doi:10.1089/thy.2011.0318 